\title{
Reconstruction and Quantification of Diffusion Tensor Imaging-Derived Cardiac Fibre and Sheet Structure in Ventricular Regions used in Studies of Excitation Propagation
}

\author{
A.P. Benson ${ }^{1}$, S.H. Gilbert, P. Li, S.M. Newton and A.V. Holden \\ Computational Biology Laboratory, Institute of Membrane and Systems Biology \\ \& Multidisciplinary Cardiovascular Research Centre, \\ Faculty of Biological Sciences, University of Leeds, Leeds LS2 9JT, UK
}

\begin{abstract}
Detailed descriptions of cardiac geometry and architecture are necessary for examining and understanding structural changes to the myocardium that are the result of pathologies, for interpreting the results of experimental studies of propagation, and for use as a three-dimensional orthotropically anisotropic model for the computational reconstruction of propagation during arrhythmias. Diffusion tensor imaging (DTI) provides a means to reconstruct fibre and sheet orientation throughout the ventricles. We reconstruct and quantify canine cardiac architecture in selected regions of the left and right ventricular free walls and the inter-ventricular septum. Fibre inclination angle rotates smoothly through the wall in all regions, from positive in the endocardium to negative in the epicardium. However, fibre transverse and sheet angles show large variability in basal regions. Additionally, regions where two populations (positive and negative) of sheet structure merge are identified. From these data, we conclude that a single DTI-derived atlas model of ventricular architecture should be applicable to modelling propagation in wedges from the equatorial and apical left ventricle, and allow comparisons to experimental studies carried out in wedge preparations. However, due to inter-individual variability in basal regions, a library of individual DTI models of basal wedges or of the whole ventricles will be required.
\end{abstract}

Key words: myocardium, anatomy, diffusion tensor imaging, anisotropy AMS subject classification: 92B15; 92C55

\footnotetext{
${ }^{1}$ Corresponding author. Email: a.p.benson@leeds.ac.uk
} 


\section{Introduction}

Fibre orientation and sheet structure throughout the myocardium, along with tissue geometry and heterogeneous cell electrophysiology, underlie many of the phenomena associated with the function of the heart, including spread of electrical excitation and mechanical properties. Propagation of electrical activity $[32,43]$ is orthotropically anisotropic, being fastest in the direction of the long axis of the fibre due to the presence of gap junctions that are principally located at the ends of the myocytes $[17,52,66]$ and slowest across the sheet plane due to the small number of muscle branches connecting otherwise electrically-insulated muscle sheets $[34,46]$. Contraction of myocytes occurs in the long axis direction and, together with transmural shear along sheet planes [16], results in transmural thickening and apex-base shortening. The fibre and sheet architecture throughout the ventricles could itself be a substrate for arrhythmias, including ventricular tachycardia and fibrillation, and sudden cardiac death, as has been suggested in both experimental $[18,48,58,60]$ and theoretical $[9,14,22]$ studies. Ventricular wedge preparations are widely used in the optical imaging of excitation during arrhythmias [21], but in order to correctly interpret the results from such wedge experiments, detailed descriptions of cardiac ventricular geometry and architecture will be required, along with an understanding of how this cardiac structure influences the propagation of excitation. Furthermore, fibre orientation can change during certain pathological conditions such as hypertrophy [25] and ischaemic heart disease [47,65], and so a complete understanding of such pathologies will require information about how cardiac geometry and architecture changes with disease.

It is therefore important to have a clear understanding of the three-dimensional architecture of the ventricles. Previous studies characterising the structure of the myocardium have utilised histological techniques in order to determine both fibre orientation $[51,55,56]$ and sheet structure [13, 46], or polarised light microscopy for determining fibre orientation [42]. Fibre orientation is known to follow a transmural helical pattern such that the inclination of the fibres with respect to the short axis of the heart (the fibre inclination angle, also referred to as the helix angle) shifts from positive at the endocardium to negative at the epicardium, changing sign at the midwall. The study of LeGrice et al. [46] suggested an organisation of the fibres at a higher level into a laminar structure with sheets approximately four myocytes thick, with these sheets separated by sheet cleavage planes. The cleavage planes ran radially from the endocardium to the epicardium and, when viewed in a long axis transmural plane, could be seen to shift from a base-apex direction near the apex through to an apex-base direction in basal regions. This architecture, however, has been disputed by several groups for numerous reasons - see [24] for a review. Furthermore, histological techniques, even if the tissue does not require fixing as for polarised light microscopy, require reconstruction of fibre and sheet orientations from sections, and introduce problems of distortion and alignment. Additionally, it is difficult to reconstruct the three-dimensional orientation of a fibre or sheet as only angles in the stack of cut planes can be directly measured.

Diffusion tensor magnetic resonance imaging (or diffusion tensor imaging, DTI) [4] has been developed as a non-destructive, high-throughput method to reconstruct in three dimen- 
sions both the fibre orientation and sheet structure throughout the ventricles. Previously applied to trace fibre tracts in the central nervous system [26, 40, 50] and study structure in cartilage [37] and skeletal muscle [3], the primary eigenvector of the diffusion tensor has been validated as a measure of cardiac fibre orientation $[33,36,53]$ and the secondary and tertiary eigenvectors have been proposed as indices [61] and validated as measures [29] of ventricular sheet structure.

Reconstructing the fibre and sheet orientation in detail throughout the ventricles allows the reconstruction of cardiac excitation using computational models [7,31]. Such wedge and whole ventricle simulations of cardiac electrophysiology are now tractable and extremely useful, as the data they provide can be dissected in time and space, and by parameters. This allows a detailed examination of the effects of cardiac structure on propagation of excitation [9], the elucidation of mechanisms underlying arrhythmias [7, 8, 15], and gives an additional means of interpreting the results from experimental studies [59]. Propagation of electrical excitation in cardiac tissue can be described by the non-linear cable equation, a reaction-diffusion-type partial differential equation:

$$
\frac{\partial V}{\partial t}=\nabla(\mathbf{D} \nabla V)-I_{\text {ion }}
$$

Here $V$ is the membrane potential in $\mathrm{mV}, t$ is time in $\mathrm{ms}, \nabla$ is a spatial gradient operator, $\mathbf{D}$ is a diffusion coefficient tensor $\left(\mathrm{mm}^{2} \mathrm{~ms}^{-1}\right)$ that characterises electrotonic spread of voltage through the tissue, and $I_{\text {ion }}$ is the total membrane ionic current density in $\mu \mathrm{A} \mu \mathrm{F}^{-1}$. Families of cardiac cell models have been developed to describe the voltage- and time-dependent current $I_{\text {ion }}$ that reconstruct the action potential $V(t)[49,57]$. These models can be applied hierarchically, with cell models for action potential properties, 1D virtual tissues for transmural propagation and vulnerability, and electrophysiologically- and anatomically-detailed 3D ventricular wedge models for intramural propagation and re-entry. The diffusion tensor $\mathbf{D}$ changes with location in the ventricles, and is determined by the tissue fibre and sheet structure at any given location. At each point in the heart there are three principal orthogonal directions of diffusion - along the fibre axis, perpendicular to the fibre axis in the sheet plane, and normal to the sheet plane - with these directions given by the orthogonal vectors $\mathbf{f}, \mathbf{s}$ and $\mathbf{n}$ respectively. The DTI dataset contains the scalar components of these three vectors with respect to a global Cartesian coordinate system. In a local coordinate system based on these orthogonal vectors, the diffusion tensor at a particular point in space is

$$
\widetilde{\mathbf{D}}=\left(\begin{array}{ccc}
D_{\|} & 0 & 0 \\
0 & D_{\perp 1} & 0 \\
0 & 0 & D_{\perp 2}
\end{array}\right)
$$

where $D_{\|}$is diffusion along the fibre axis, $D_{\perp 1}$ is diffusion in the sheet plane perpendicular to the fibre axis and $D_{\perp 2}$ is diffusion normal to the sheet plane (i.e. in the orthogonal directions $\mathbf{f}, \mathbf{s}$ and $\mathbf{n}$ respectively). As the three vectors $\mathbf{f}, \mathbf{s}$ and $\mathbf{n}$ are the eigenvectors of the diffusion tensor $\mathbf{D}$ with corresponding eigenvalues $D_{\|}, D_{\perp 1}$ and $D_{\perp 2}$, then the transformation matrix of $\widetilde{\mathbf{D}}$ to $\mathbf{D}$ is an orthogonal matrix $\mathbf{A}=(\mathbf{f}, \mathbf{s}, \mathbf{n})$. The diffusion tensor $\mathbf{D}$ is therefore given 
by $\mathbf{D}=\mathbf{A} \widetilde{\mathbf{D}} \mathbf{A}^{\mathrm{T}}$, and substitution gives

$$
\mathbf{D}=D_{\|} \mathbf{f f}^{\mathrm{T}}+D_{\perp 1} \mathbf{s s}^{\mathrm{T}}+D_{\perp 2} \mathbf{n} \mathbf{n}^{\mathrm{T}} .
$$

Histologically-derived digital datasets describing geometry and architecture (and thus the diffusion tensor $\mathbf{D}$ in Equation 0.1) in canine [38, 39, 45] and rabbit [63] hearts are spatially smoothed models. However, recent studies suggest that sheet structure is highly variable between individual canines, and is variable between species [24]. As such, a single structural model may not be appropriate for simulating propagation in an anatomically detailed ventricular wedge, or in the complete ventricles. DTI is a method that can be used to build libraries of fibre and sheet structure highlighting the intra- and inter-species variation in myocardial architecture in both physiological and pathological states. Such libraries would provide geometries on which to run normal, pathological and pharmacologically-modified biophysically-detailed excitation equations and further understand the process of excitation propagation in the myocardium and the mechanisms underlying cardiac arrhythmias.

Any dataset used to describe the diffusion tensor D in Equation 0.1 must be validated that is, the vectors contained within the dataset must correspond to the actual fibre and sheet structure throughout the myocardium. Here we present an extraction and quantification of myocardial architecture from three regions commonly used in wedge optical imaging studies and computational simulations (basal, equatorial and apical lateral left ventricle), using data from three canine ventricles imaged using DTI, along with a description of the methods used to extract and analyse these data. We reconstruct and analyse the transmural course of the fibre inclination and transverse angles and the sheet angle, and investigate the degree of variability in these angles.

\section{Methods}

\subsection{Diffusion Tensor Imaging Datasets}

Three mongrel dog hearts were imaged using DTI, and subsequently pre-processed, by Professor Edward Hsu and colleagues, then at the Center for In Vivo Microscopy, Duke University Medical Center. Acquisition of the DTI data was a whole-ventricle analogue of the technique described in detail previously [35, 36]. Tissue geometry from an MRI file was used to mask the DTI data so that the tensors of voxels lying within the ventricular tissue could be isolated from those lying outside. The three orthogonal eigenvectors and corresponding eigenvalues of the diffusion tensor were then calculated at each voxel throughout the ventricular tissue, with the primary eigenvector having the largest eigenvalue and the tertiary eigenvector the smallest.

\subsection{Coordinate System}

In order to reconstruct fibre and sheet orientation throughout the ventricles, the Cartesian coordinate system of the DTI data was converted to a cylindrical coordinate system (see 
Figure 1). The eigenvectors were first reoriented with respect to the long axis of the heart within the anatomical coordinate system, which differed from the $\mathrm{z}$ axis of the DT imaging coordinates (Figure 1a). This new apex-base axis was fit to the centre of the left ventricle of each heart. The centroid of the polar coordinate system was given by the intersection of this apex-base axis with the slice, and a radial axis for each voxel defined as a line perpendicular to the apex-base axis connecting the centroid and the voxel. A third axis, the tangential axis, lies perpendicular to both the apex-base and radial axes (Figure 1b). For any particular location within the three-dimensional space we then defined three orthogonal reference planes: i) the transverse plane is approximately the short axis of the heart, with the apex-base axis normal to this plane; ii) the circumferential plane lies approximately tangential to the epicardial surface, with the radial axis normal to this plane; and iii) the radial plane, which is perpendicular to the transverse and tangential planes and passes through the centroid and the voxel. Fibre and sheet angles were calculated with respect to these three reference planes (see Figure 2).

(a)

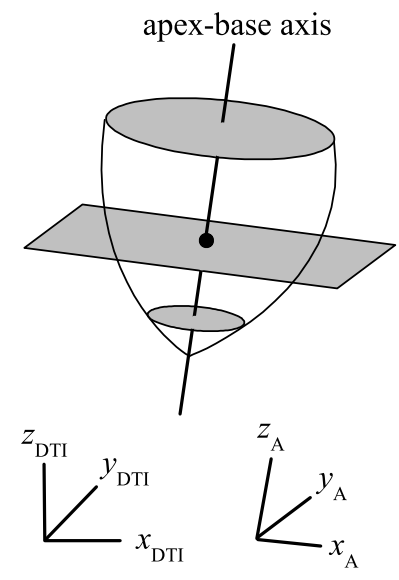

(b)

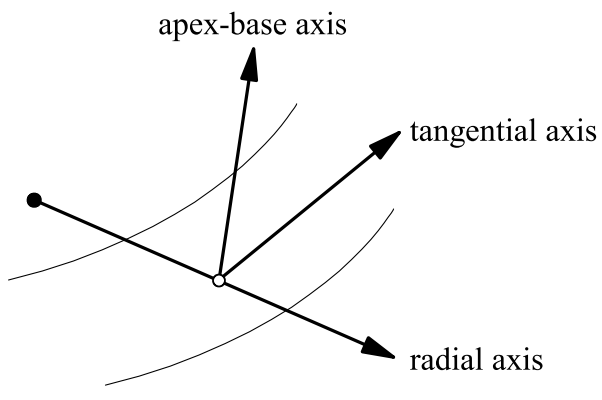

Figure 1: Coordinate system used to describe fibre and sheet orientations in the heart. (a) A base-apex axis is fit to the centre of the left ventricle. This axis is normal to the transverse plane, shown in grey. The centroid for each slice is the location where the apex-base axis intersects the transverse plane, and is shown as a filled circle. The subscript DTI denotes the diffusion tensor coordinate system, the subscript A denotes the new anatomical coordinate system. (b) For each voxel (shown here as an open circle), three orthogonal reference axes are defined. The base-apex axis is parallel to that defined previously, the radial axis passes through the centroid and the voxel, and the tangential axis lies in the transverse plane perpendicular to the other two axes. 
(a)

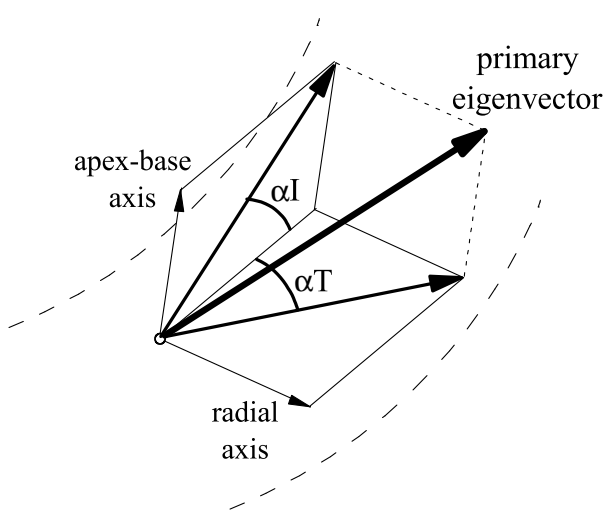

(b)

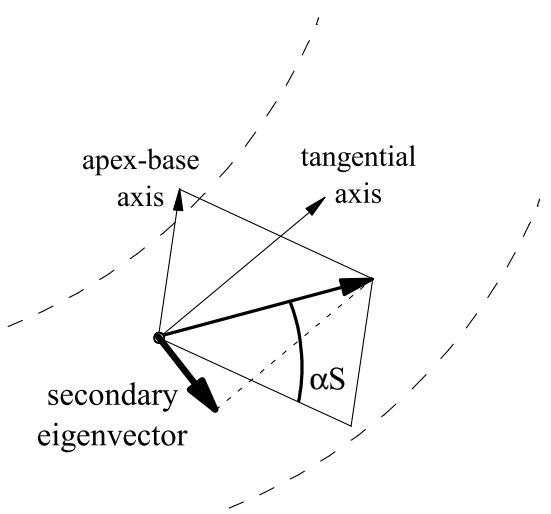

Figure 2: Definition of angles. (a) The fibre inclination angle $(\alpha \mathrm{I})$ and the fibre transverse angle $(\alpha \mathrm{T})$. (b) The sheet angle $(\alpha \mathrm{S})$. Axes are as defined in Figure 1.

\subsection{Selection of Slices and Sectors}

Since each of the DTI datasets contains approximately $10^{6}$ voxels, we analysed extracted data from sectors at specific locations. For each heart we chose three representative slices. The basal slice was the first slice that did not show any valves or great vessels. The apical slice was chosen to be the slice nearest the apex that showed the chamber of the left ventricle. The equatorial slice was chosen to be as close to midway between the basal and apical slices as possible, with the additional constraint that the chamber of the right ventricle, and therefore the right ventricular free wall (RVFW), were visible. For each selected slice, we then chose five $15^{\circ}$ wide sectors from which to extract data (see Figure 3 ). Anterior and posterior sectors in the left ventricular free wall (LVFW) were located between the RVFW fusion sites and the respective left ventricle papillary muscles, while the LVFW lateral sector was located in the inter-papillary muscle region of the left ventricle. The septal sector was located between the two RVFW fusion sites. The RVFW sector (basal and equatorial slices only) took the same orientation as the septal sector. In all cases, the location of the sector was chosen to minimise inclusion of papillary muscle. If voxels containing papillary muscle were included within a sector, these were digitally removed.

In addition to the sectors from which angles were quantified, regions where abrupt changes in sheet angle occurred, from positive to negative or vice-versa, were identified in order to allow the reconstruction of the fibre orientation and sheet plane across such transitions. These transition regions were located approximately equatorially, although because of the variable locations of such regions [24] they did not necessarily coincide with the slices selected for quantification of angles - see Results for more details. 


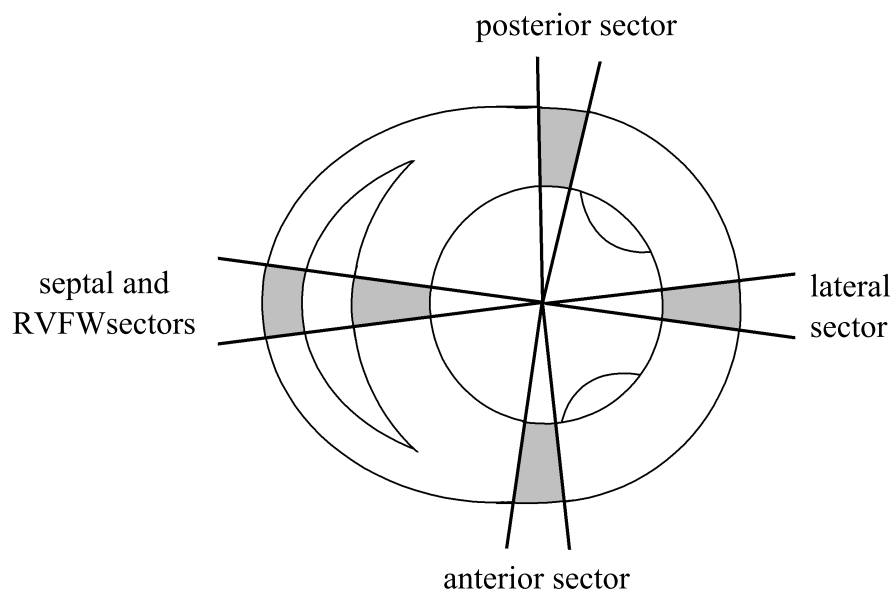

Figure 3: Sectors from which fibre and sheet angles were extracted in each transverse slice. See text for details.

\subsection{Normalised Transmural Position}

A normalised transmural position was assigned to each voxel in each sector using the method described by Geerts et al. [23]. The location of the midwall was first defined as the position where a fit to the transmural course of the inclination angles (see definition below) changed sign. The radius from the centroid to the midwall in each sector was defined as the distance $R_{0}$. Normalised transmural position, $h$, was then specified using $h=\left(R-R_{0}\right) / R_{0}$, where $R$ is the distance of the voxel from the centroid. In order to compensate for the larger distance of the RVFW midwall from the centroid, the transmural position in the RVFW sector is given by $h=\left(R-R_{0}\right) / R_{0 \mathrm{~S}}$, where $R_{0 \mathrm{~S}}$ is the midwall location in the septal sector. Thus, data from different hearts were aligned at the midwall where $h=0$, and the centroid for each slice is at $h=-1$. This normalisation method removes errors associated with identifying the locations of the endocardial and epicardial surfaces and has been validated by Geerts et al. [23], who showed that the standard deviation of the inclination angle data was much reduced when compared to the usual endocardial-to-epicardial normalisation method.

\subsection{Fractional Anisotropy}

Fractional anisotropy (FA) is a measure of the anisotropy of diffusion at a particular voxel within the tissue (i.e. whether the fibre direction can be well determined), and was calculated at each voxel throughout the tissue using

$$
\begin{gathered}
\mathrm{FA}=\sqrt{\frac{3}{2} \cdot \frac{\left(\lambda_{1}-\langle\lambda\rangle\right)^{2}+\left(\lambda_{2}-\langle\lambda\rangle\right)^{2}+\left(\lambda_{3}-\langle\lambda\rangle\right)^{2}}{\lambda_{1}^{2}+\lambda_{2}^{2}+\lambda_{3}^{2}}}, \\
\langle\lambda\rangle=\frac{\lambda_{1}+\lambda_{2}+\lambda_{3}}{3}
\end{gathered}
$$


where $\lambda_{1}, \lambda_{2}$ and $\lambda_{3}$ are the primary, secondary and tertiary eigenvalues respectively [6, 44]. A value of $\mathrm{FA}=0$ indicates isotropy of diffusion (i.e. the magnitude of diffusion is the same in the fibre axis, sheet plane and sheet normal directions such that $\lambda_{1}=\lambda_{2}=\lambda_{3}$ ), while a value of $\mathrm{FA}=1$ indicates cylindrically symmetrical anisotropy where $\lambda_{1} \gg \lambda_{2}=\lambda_{3}$.

\subsection{Fibre and Sheet Angles}

For any particular fibre orientation the eigenvector can point in one of two directions, each lying along the long axis of the myocyte but at $180^{\circ}$ to each other. We calculated the angle between the tangential axis and the projection of the primary eigenvector onto the transverse plane (which lies in the range $\left[0^{\circ}: 180^{\circ}\right]$ ), and flipped the orientation of the vector if this angle was $\geq 90^{\circ}$. Thus the projections of all primary eigenvectors onto the transverse plane formed angles less than $90^{\circ}$ with the tangential axis, and pointed in an anticlockwise direction when viewed from a base to apex direction. The fibre inclination angle was defined as the angle between the transverse plane and the projection of the primary eigenvector onto the circumferential plane (see Figure 2a). Taking this angle rather than the direct angle between the eigenvector and the transverse plane allows comparisons with data obtained using histological methods, which are generally taken from slices made tangential to the epicardial surface.

Fibre orientation can only be fully described by the inclusion of a second angle showing the deviation of the fibre on the transverse plane. This transverse angle is given as the angle between the circumferential plane and the projection of the fibre onto the transverse plane (see Figure 2a).

If the secondary and tertiary eigenvalues statistically belong to distinct populations, then diffusivity within the tissue is transversely anisotropic (i.e. orthotropically ansotropic). The secondary and tertiary eigenvectors then provide measures of sheet plane and sheet normal directions, respectively. We used the statistical method developed by Helm et al. [29] to determine whether the secondary and tertiary eigenvalues do belong to distinct populations. For each sector in each heart a test statistic, $T$, was calculated using

$$
T=\frac{\operatorname{Mdn}(\xi)}{\operatorname{Mdn}(|\xi-\operatorname{Mdn}(\xi)|)},
$$

where $\operatorname{Mdn}(x)$ indicates the median value of $x$, and $\xi=\lambda_{2}-\lambda_{3}$. The critical value, $T_{\mathrm{C}}$, that the test statistic had to reach to be significant at the $1 \%$ level was dependent on the number of voxels in the sector, $n$, and was calculated as

$$
T_{\mathrm{C}}=\frac{9.0}{\sqrt{n}}+2.53
$$

where the constants 9.0 and 2.53 were obtained from Monte Carlo simulations in which $T_{\mathrm{C}}$ was determined from distributions of eigenvalues of varying sample size [29]. In all sectors examined in all hearts, $T>T_{\mathrm{C}}$ (see Table 1), and so we assumed transverse anisotropy of diffusion and extracted sheet angles from the sectors. We took the secondary eigenvector to 
Table 1: Test values, $T$, and critical test values, $T_{\mathrm{C}}$, to determine distinct populations of secondary and tertiary eigenvalues in lateral sectors from basal, equatorial and apical slices in the three canine hearts. Asterisks indicate $T>T_{\mathrm{C}}$ and so a significant difference $(P<0.01)$ exists between the eigenvalues.

\begin{tabular}{cccc}
\hline Slice & Heart & $T_{\mathrm{C}}$ & $T$ \\
& & & \\
\hline \multirow{2}{*}{ Basal } & $\mathrm{A}$ & 3.08 & $4.63^{*}$ \\
& $\mathrm{~B}$ & 3.10 & $5.18^{*}$ \\
& $\mathrm{C}$ & 3.06 & $5.52^{*}$ \\
& & & \\
Equatorial & $\mathrm{A}$ & 2.94 & $2.97^{*}$ \\
& $\mathrm{~B}$ & 3.07 & $7.93^{*}$ \\
& $\mathrm{C}$ & 3.05 & $4.92^{*}$ \\
Apical & $\mathrm{A}$ & 3.16 & $7.44^{*}$ \\
& $\mathrm{~B}$ & 3.02 & $6.11^{*}$ \\
& $\mathrm{C}$ & 3.28 & $6.30^{*}$ \\
\hline
\end{tabular}

indicate the orientation of the sheet plane. As with the primary eigenvector, the secondary eigenvector can take one of two directions, both being perpendicular to the local fibre orientation and lying in the sheet plane but at $180^{\circ}$ to each other. In the case of sheet structure, we flipped the secondary eigenvector if the angle formed by the radial axis and the projection of the secondary eigenvector onto the transverse plane was $\geq 90^{\circ}$. Thus all secondary eigenvectors pointed away from the centroid, approximately in an endocardial to epicardial orientation. Secondary eigenvectors in the heart point principally in the radial direction $[20,41,53,54]$ and so we calculate the sheet angle as the angle between the transverse plane and the projection of the secondary eigenvector onto the radial plane (see Figure $2 \mathrm{~b}$ ). This also allows comparisons to data from histological studies [11, 45, 46] which are generally taken from slices made in the radial plane.

\subsection{Statistical Analysis}

Results are presented as mean \pm standard deviation. Significant differences between means of groups were tested for using independent $t$ tests, or one-way analysis of variance (ANOVA) with Tukey post hoc tests. Values of $P<0.05$ were considered statistically significant. 
Table 2: Wall thickness $(\mathrm{mm})$ in the three canine hearts.

\begin{tabular}{lccccr}
\hline & LVFW & Apical LVFW & Basal LVFW & Septum & RVFW \\
& & & & & \\
\hline Heart A & $16.4 \pm 3.1$ & $16.3 \pm 2.9$ & $16.6 \pm 3.4$ & $16.5 \pm 3.6$ & $7.6 \pm 3.1$ \\
Heart B & $16.0 \pm 5.6$ & $16.0 \pm 6.3$ & $15.9 \pm 3.6$ & $15.2 \pm 4.6$ & $7.3 \pm 2.3$ \\
Heart C & $15.8 \pm 4.2$ & $15.2 \pm 4.1$ & $17.1 \pm 4.1$ & $14.9 \pm 3.9$ & $11.2 \pm 4.7$
\end{tabular}

\section{Results}

\subsection{Ventricular Geometry}

Ventricular geometries of the three hearts are reconstructed and visualised in Figure 4, showing the endocardial and epicardial surfaces, trabeculae carnae and papillary muscles. The boundaries of the papillary muscles are blurred by temporal averaging of in vivo MRI of the beating heart, and are not apparent in the Auckland canine heart model [38, 39, 45]. All three hearts showed anterior and posterior papillary muscles in the left ventricles. In the right ventricle, two hearts showed anterior, septal and posterior papillary muscles, while the third heart showed only anterior and posterior papillary muscles, although the anterior muscle was somewhat larger compared to the other hearts. Trabeculae and papillary muscles may act as initiation and/or pinning sites for re-entrant waves during ventricular fibrillation [62, 64] and so digital models of cardiac geometry that are to be used in the simulation of propagation and arrhythmias need to include the details of endocardial structures. Ventricular wall thickness in the entire LVFW, the apical and basal portions of the LVFW, and in the RVFW is shown in Table 2. LVFW thickness is significantly lower in apical regions compared to basal regions in two of the hearts. In heart A, apical and basal LVFW thicknesses were $16.3 \pm 2.9$ and $16.6 \pm 3.4 \mathrm{~mm}$ respectively $(t=3.76, P<0.05)$ and in heart $\mathrm{C}$ were $15.2 \pm 4.1$ and $17.1 \pm 4.1$ $\mathrm{mm}(t=24.61, P<0.05)$. There was no significant difference between the apical and basal LVFW thickness in heart B $(16.0 \pm 6.3$ and $15.9 \pm 3.6 \mathrm{~mm})$. Ventricular chamber volumes are shown in Table 3.

We measured FA values of $0.24,0.24$ and 0.28 in hearts A, B and C respectively, each with the standard deviation of 0.09. Our mean value for the three combined hearts of $\mathrm{FA}=0.25 \pm 0.09$ is similar to measurements from other studies, with values of 0.36 in canine [29], 0.35 in goat [23], 0.27 in mouse [41], and $0.36,0.32$ and 0.3 in diastolic, early and late systolic rat respectively [11] having been reported. Thus, fibre direction in the three hearts was well determined.

Fibre inclination angle, fibre transverse angle and sheet angle maps for the cut surfaces of slabs digitally dissected from the three hearts are shown in Figure 5. Fibre inclination 

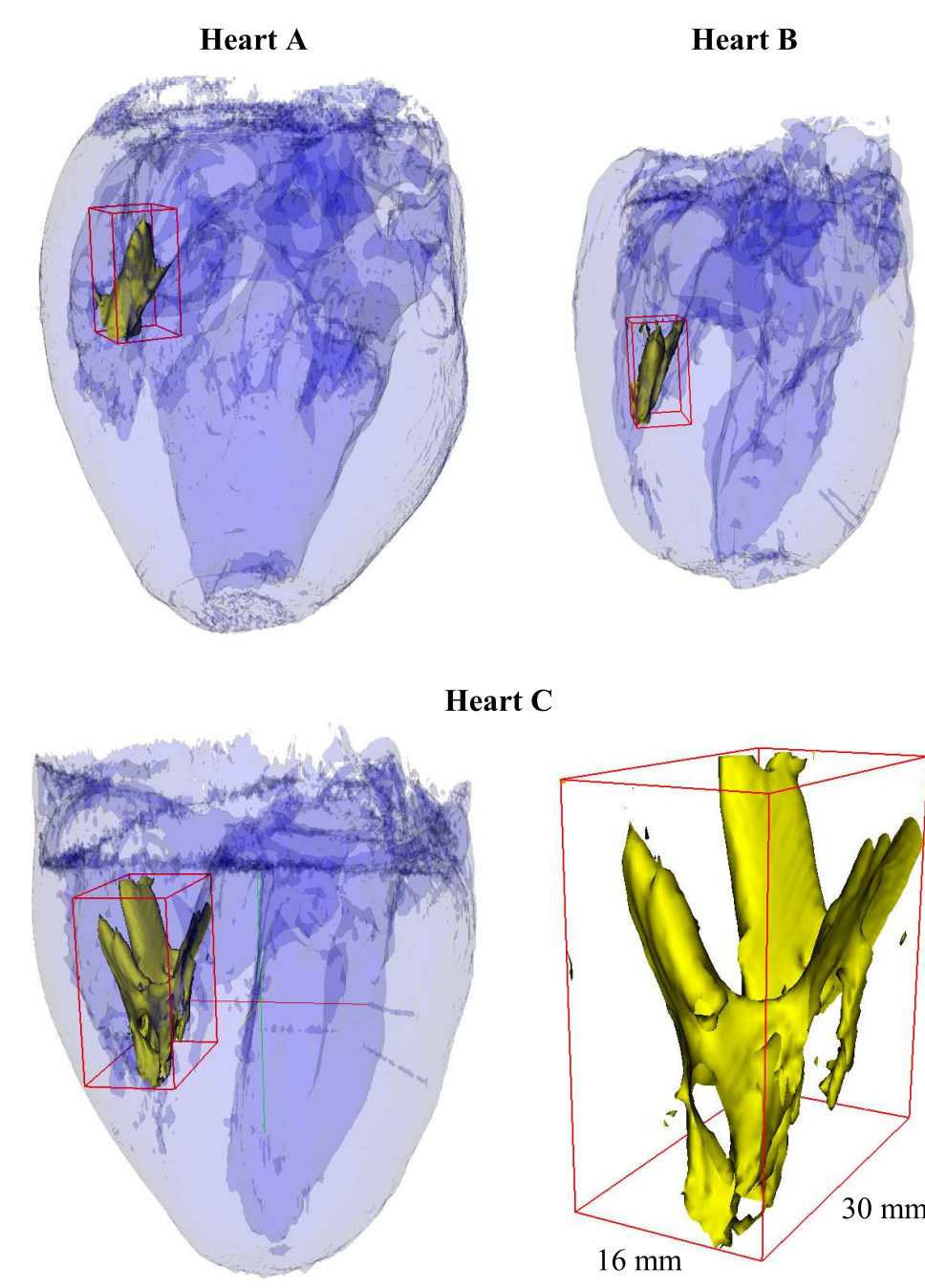

Heart C

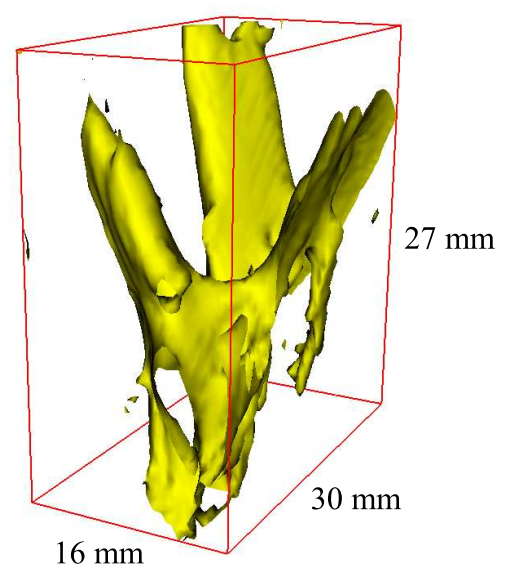

Figure 4: Visualisation of reconstructed ventricular geometry from three canine hearts, with endocardial surfaces shown in dark blue and epicardial surfaces in light blue. The resolution of each voxel is $390 \times 780 \times 780 \mu \mathrm{m}$, sufficient to visualise trabeculae and papillary muscles. The box in the right ventricle of each heart highlights the papillary muscles, which are extracted and enlarged from heart C. 
Table 3: Ventricular chamber volumes $(\mathrm{ml})$ in the three canine hearts. LV, left ventricle; $\mathrm{RV}$, right ventricle.

\begin{tabular}{llll}
\hline & LV & RV & LV $/$ RV \\
& & & \\
\hline Heart A & 71.64 & 52.39 & 1.37 \\
Heart B & 22.98 & 30.40 & 0.76 \\
Heart C & 46.84 & 55.09 & 0.85 \\
& & & \\
\hline
\end{tabular}

and transverse angles give a measure of the direction of anisotropy within a particular tissue sheet. The fibre inclination angle shows a clear endocardial-to-epicardial angle change of $\sim 120^{\circ}$ in the LVFW, inter-ventricular septum and RVFW, from positive at the endocardial surface to negative at the epicardial surface, changing sign in the midwall (Figure 5a). The sheet angles, which together with the fibre orientation provide a measure of the principal directions of tissue orthotropy, generally appear to be positive in the LVFW (Figure 5c).

\subsection{Fibre Inclination Angle}

Figure 6 shows fibre inclination angle in apical, equatorial and basal slices of the three hearts, with the shading indicating the absolute value of the angle using a grey scale, such that white shows fibres lying in the transverse plane and black shows fibres normal to this plane. The transmural change from dark to light then to dark again is clearly evident in all slices, consistent with an endocardial-to-epicardial rotation of $\sim 120^{\circ}$. Also striking is the integrity of the left ventricle, which in all slices of all three hearts appears as a continuous ring (or cone in three dimensions) of tissue composed of the LVFW and the inter-ventricular septum. In visualisations of the fibre inclination angle, the RVFW appears to be an attachment to this ring of tissue.

To allow examination of the consistency in the transmural change of the fibre inclination angles in regions of the heart, these angles were quantified for sectors in equatorial slices and are plotted against normalised transmural position in Figure 7. This normalisation of transmural position aligns data from different hearts at the centre of the left ventricle chamber (normalised transmural position $=-1$ ) and at the midwall (normalised transmural position $=0$ ); the normalised locations of the endocardial and epicardial surfaces from a particular heart are therefore determined by the relative sizes of the left ventricular chamber and the LVFW, inter-ventricular septum or RVFW thickness. Linear fits to data from individual hearts quantifies the transmural course of the angle.

According to these linear fits, the endocardial-to-epicardial course of the fibre inclination 
(a)
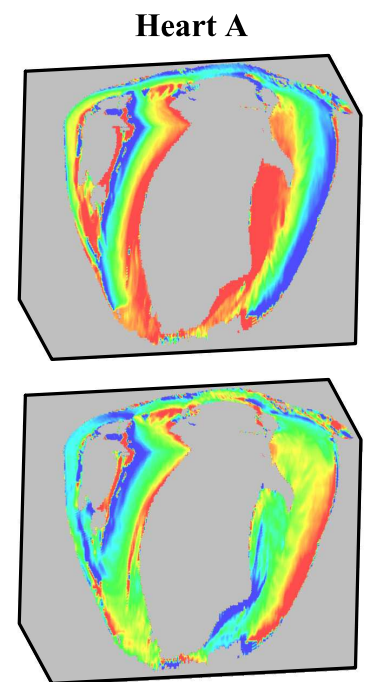

(c)
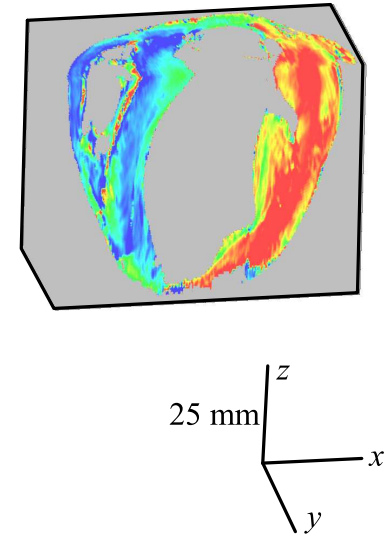

Heart B

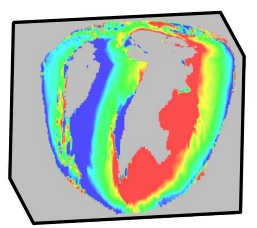

Heart C
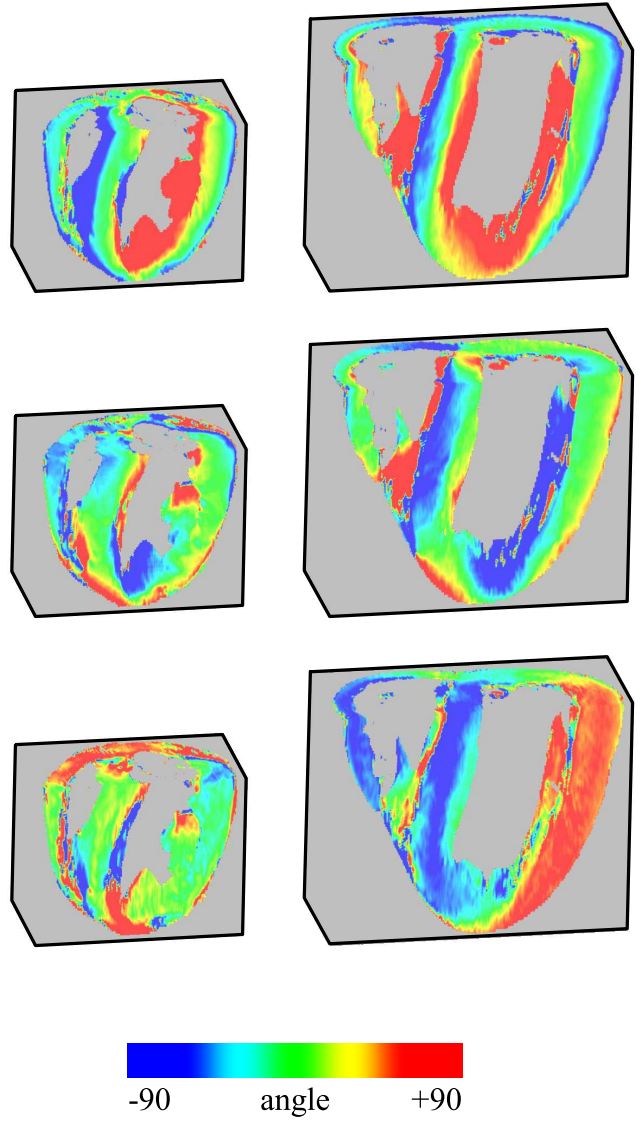

Figure 5: Reconstructed fibre inclination angle (a), fibre transverse angle (b) and sheet angle (c) maps on the cut surface of slabs digitally dissected from the three hearts. Each slab was cut in the short axis plane to show the right and left ventricular chambers, and in the longitudinal plane to show the right and left ventricular free walls and the inter-ventricular septum. 


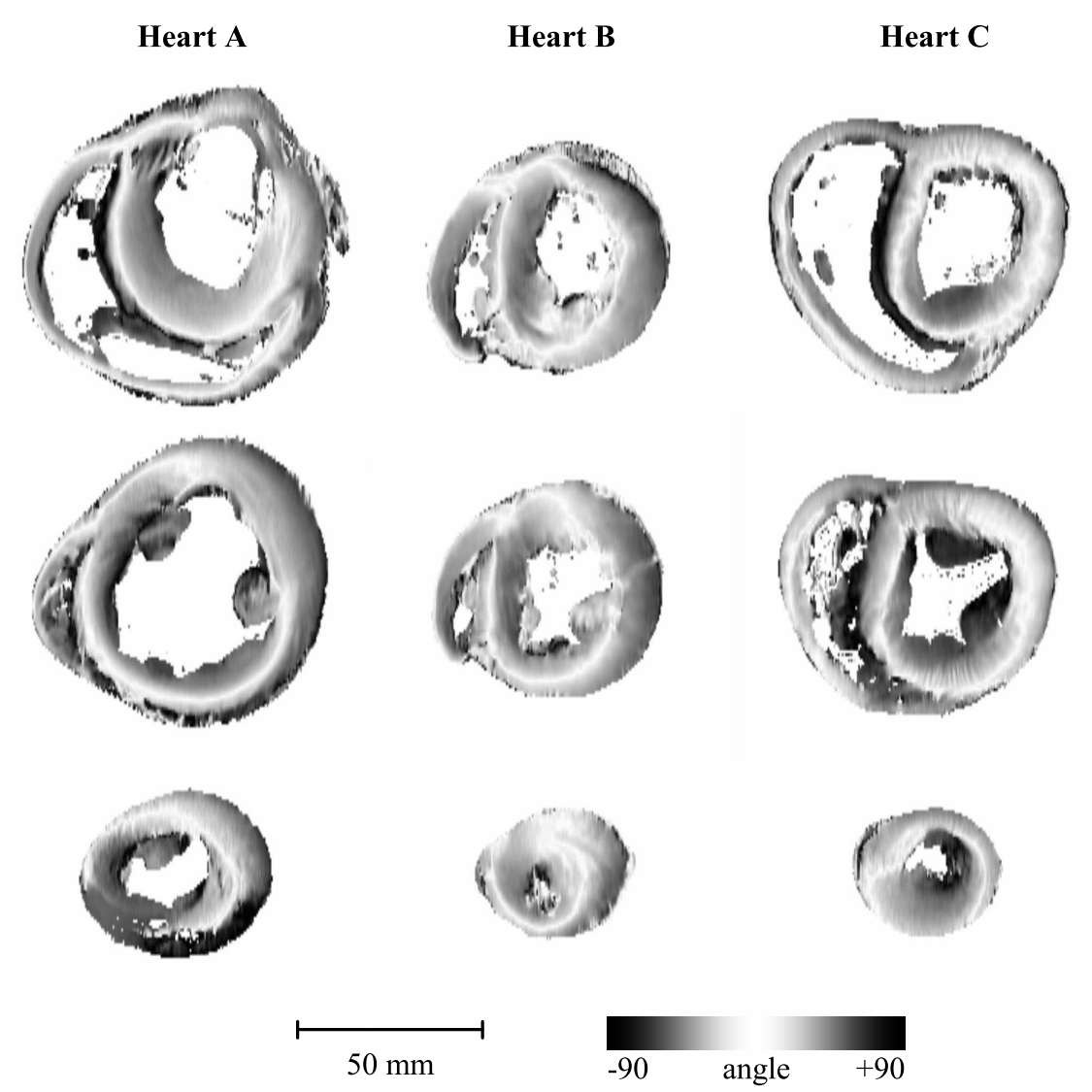

Figure 6: Fibre inclination angles in short axis basal (top), equatorial (middle) and apical (bottom) slices through three canine hearts. The shading shows the absolute value of the fibre inclination angle using a grey scale, such that white indicates fibres lying in the transverse plane and black indicates fibres normal to this plane. 

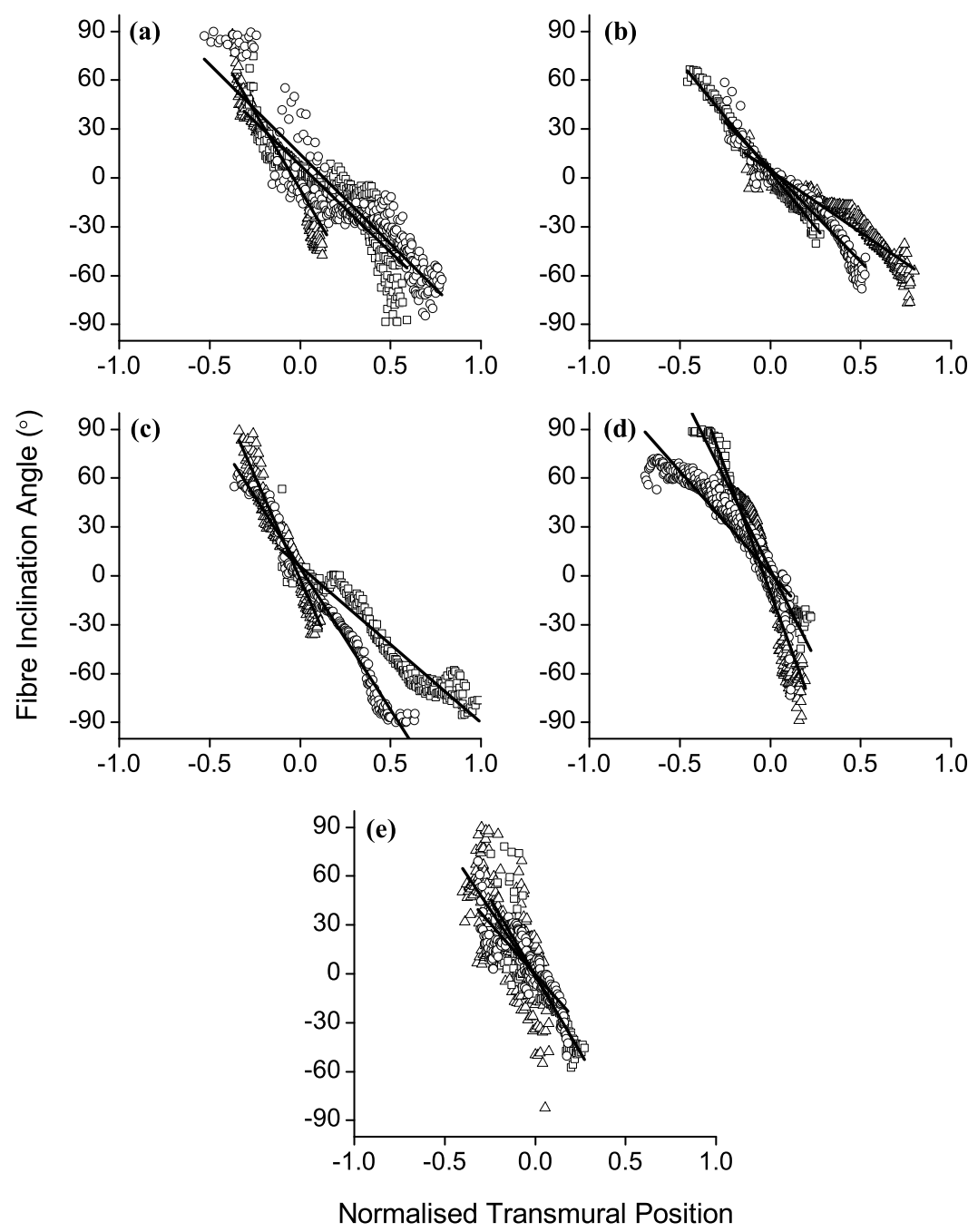

Figure 7: Transmural course of the fibre inclination angle in hearts A (triangles), B (squares) and C (circles) extracted from posterior, lateral, septal, anterior and RVFW sectors of equatorial slices (panels a to e respectively). Solid lines show linear fits to data from individual hearts. 
Table 4: Range of fibre inclination angles in equatorial slice sectors of the three hearts. The values in different sectors are not significantly different

\begin{tabular}{crrrrr}
\hline & Anterior & Lateral & Posterior & Septal & RVFW \\
& & & & & \\
\hline Heart A & 158.4 & 71.3 & 98.8 & 113.5 & 79.1 \\
Heart B & 145.6 & 100.0 & 96.3 & 104.8 & 98.2 \\
Heart C & 100.6 & 89.2 & 144.0 & 174.0 & 62.6 \\
Mean & 134.9 & 86.8 & 113.0 & 130.8 & 80.0 \\
SD & 30.4 & 14.5 & 26.8 & 37.7 & 17.8 \\
& & & & & \\
\hline
\end{tabular}

angle in combined data from the hearts changes from $+90^{\circ}$ to $-69^{\circ}$ in the anterior sector, $+66^{\circ}$ to $-56^{\circ}$ in the lateral sector, $+73^{\circ}$ to $-72^{\circ}$ in the posterior sector, $+83^{\circ}$ to $-90^{\circ}$ in the septal sector and $+88^{\circ}$ to $-53^{\circ}$ in the RVFW sector. In the endocardial region of the anterior sector, the inclination angle shows a plateau at approximately $+70^{\circ}$, and the septal sector shows a plateau at around $-70^{\circ}$ in the endocardial region of the right ventricle. The ranges of the fibre inclination angles in individual hearts are quantified in Table 4 . The mean range was smallest in the lateral sector at $112.1^{\circ}$ and largest in the posterior sector at $161.2^{\circ}$, but values were not significantly different between sectors $(F=1.35, P=\mathrm{NS})$. The slopes of the transmural course of the fibre inclination angles in individual hearts are quantified in Table 5. The mean slope was lowest in the lateral sector $\left(-91.5^{\circ}\right)$ and greatest in the anterior sector $\left(-239.7^{\circ}\right)$, but values were not significantly different between the sectors $(F=1.37$, $P=N S)$.

\subsection{Fibre Transverse Angle}

The transmural course of the fibre transverse angle is shown for basal, equatorial and apical lateral sectors in Figure 8 with linear fits for data from individual hearts. In the apical sector the fibre transverse angle shows a transmural change from approximately $-57^{\circ}$ at the endocardial surface through to $+63^{\circ}$ at the epicardial surface, according to the linear fits. In the equatorial sector this range is from $-45^{\circ}$ to $+32^{\circ}$. In the basal sector the fibre transverse angle shows negative slopes in heart A (triangles) and B (squares), and a positive slope in heart $\mathrm{C}$ (circles), although there is great variability within the data. This general trend is characteristic of the fibre transverse angle in the other sectors from the basal slices (results not shown). 
Table 5: Slope of the transmural course of fibre inclination angles in equatorial slice sectors of the three hearts. The values in different sectors are not significantly different.

\section{Anterior Lateral Posterior Septal RVFW}

$\begin{array}{crrrrr}\text { Heart A } & -304.8 & -75.9 & -190.4 & -251.1 & -165.1 \\ \text { Heart B } & -222.1 & -136.3 & -107.0 & -96.1 & -189.5 \\ \text { Heart C } & -125.0 & -114.8 & -110.2 & -174.4 & -126.5 \\ \text { Mean } & -217.3 & -109.0 & -135.9 & -173.9 & -160.4 \\ \text { SD } & 90.0 & 30.6 & 47.3 & 77.5 & 31.8\end{array}$

(a)

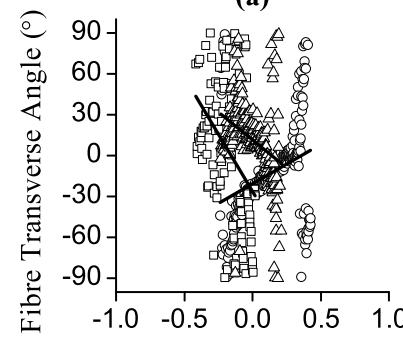

(b)

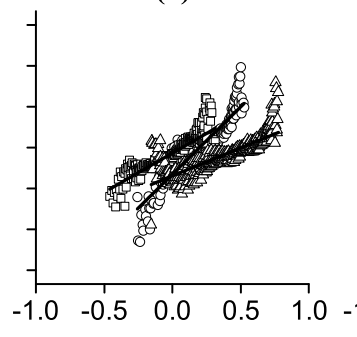

Normalised Transmural Position (c)

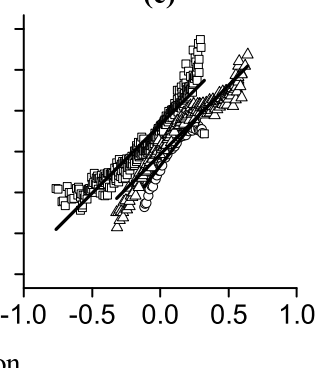

Figure 8: Transmural course of the fibre transverse angle extracted from the lateral sectors of hearts A (triangles), B (squares) and C (circles) in basal, equatorial and apical slices (panels a to $\mathrm{c}$ respectively). Solid lines show linear fits to data from individual hearts. 


\subsection{Sheet Angle}

Sheet angles as a function of transmural position are shown for the lateral sectors from apical, equatorial and basal slices in Figure 9 with linear fits for data from individual hearts. The majority of transmural locations in the three sectors from all three hearts show positive sheet angles, except in the subendocardial region of the basal sector in heart A (triangles, Figure 9a) and the subepicardial region of the apical sector in the same heart (triangles, Figure 9c). For any given transmural location, there is significant inter-heart variability in the sheet angle in apical and especially basal lateral sectors. The transmural course of the sheet plane is reconstructed in Figure 10 for individual hearts, and for the data from all three hearts combined, from 5th order polynomial fits [23] applied to the data in Figs. 7 and 9. As the sheet angles are positive for the majority of transmural locations in the sectors then the sheets are orientated in an apex-base direction for the three sectors from the each of the three hearts, and also for the combined data. Exceptions occur in the subepicardial regions of the apical sectors in heart A and the combined data, and the subendocardial regions in the basal sector of heart A, where the sheet angles are negative and the sheet planes are therefore orientated in a base-apex direction. Note the large inter-heart variability in the transmural course of the sheet plane in basal regions and, to a lesser extent, in apical regions, corresponding to the variation in the quantified angles shown in Figure 9.

(a)

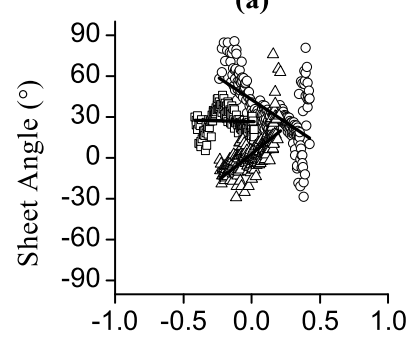

(b)

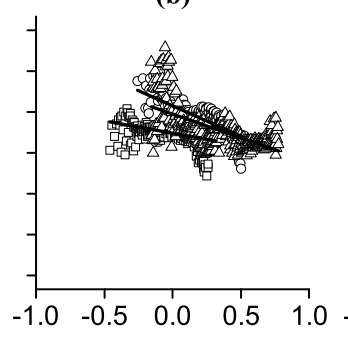

Normalised Transmural Position (c)

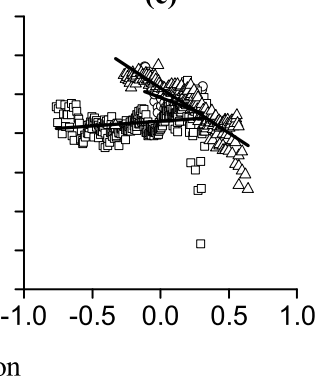

Figure 9: Transmural course of the sheet angle extracted from the lateral sectors of hearts A (triangles), B (squares) and C (circles) in basal, equatorial and apical slices (panels a to c respectively). Solid lines show linear fits to data from individual hearts.

Although sheet behaviour is qualitatively similar in the selected lateral regions (that is, mainly orientated in an apex-base direction), Figure 5c shows that, in other cardiac regions, sheet orientation varies markedly between the hearts. The posterior wall of hearts $\mathrm{A}$ and $\mathrm{C}$ has planar sheet orientation (i.e. a sheet angle close to zero such that the sheets lie in the transverse plane) while in the same region of heart B sheets are negatively orientated. Indeed, longitudinal (base-apex) columns of qualitatively similar sheet orientations can be identified. In some regions, such as the septum in heart $\mathrm{C}$, these columns of positive and negative sheet angles merge, with this merging region occurring at variable transmural and circumferential 

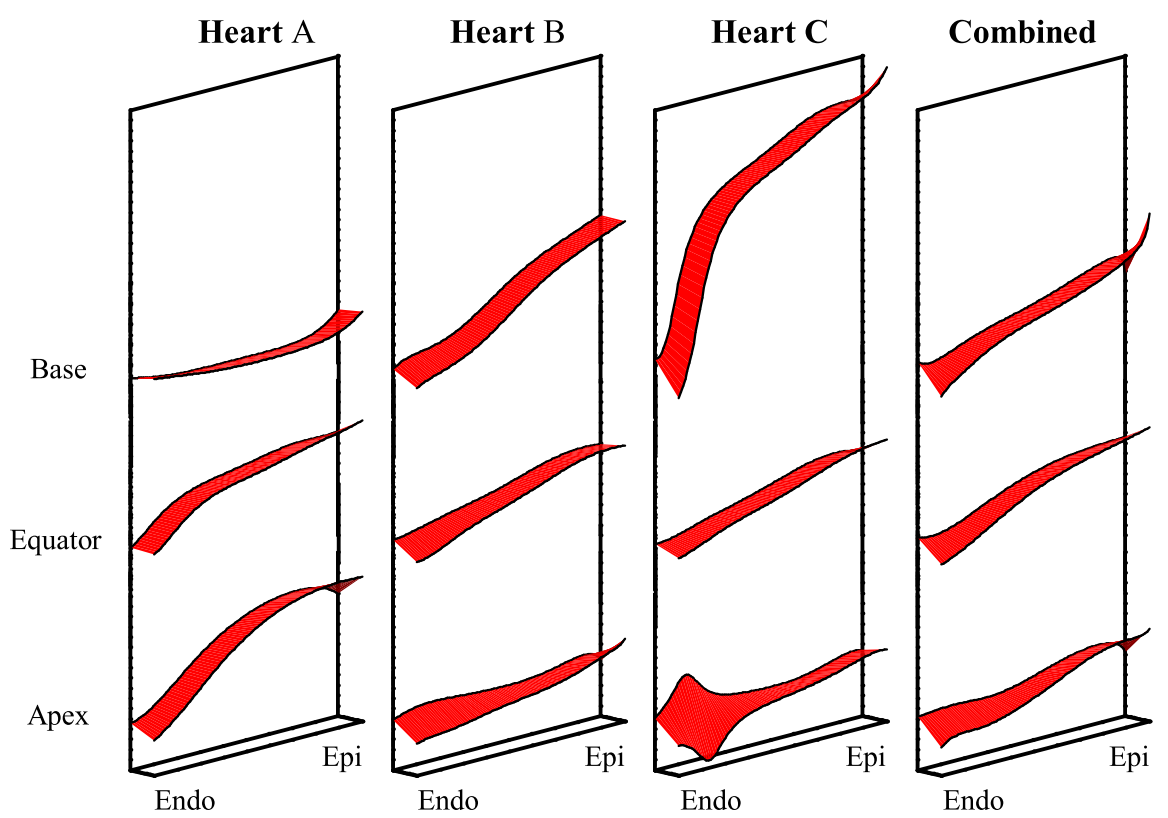

Figure 10: Reconstructions of the transmural course of the sheet plane in lateral sectors of basal, equatorial and apical slices. The elevation of the sheet plane was calculated by integrating 5th order polynomials fitted to the data from individual hearts and to all data combined (data shown in Figure 9). The twist in the sheet plane represents fibre orientation and is given by 5 th order polynomial fits to the fibre inclination angles from individual hearts, and the combined fibre inclination angles, extracted from the lateral sectors of the respective slices (shown for the lateral sector of the equatorial slice in Figure 7b). 
locations (see [24] for a review). The nature of these regions of sheet orientation transition is explored in Figure 11. Regions were identified from each heart where sheet angle transitions occur abruptly, from positive to negative or vice versa - the location and morphology of these transitions was variable (Figure 11a). The sheet angle was visualised in slabs spanning these transition regions, and the three-dimensional orientation of the primary eigenvector (fibre orientation) was visualised transmurally across the slabs. The fibres rotate smoothly, and the rotation is not affected by the abrupt sheet angle transition (Figure 11b). In Figure 11c, the three-dimensional sheet plane arrangement for the heart $\mathrm{C}$ sheet transition zone in the septal region is reconstructed using 5th order polynomial fits applied to the fibre inclination and sheet angles (as for Figure 10). This reveals an abrupt deflection of approximately $70^{\circ}$ in the sheet plane, corresponding to the sharp change in sheet angle across this transition.

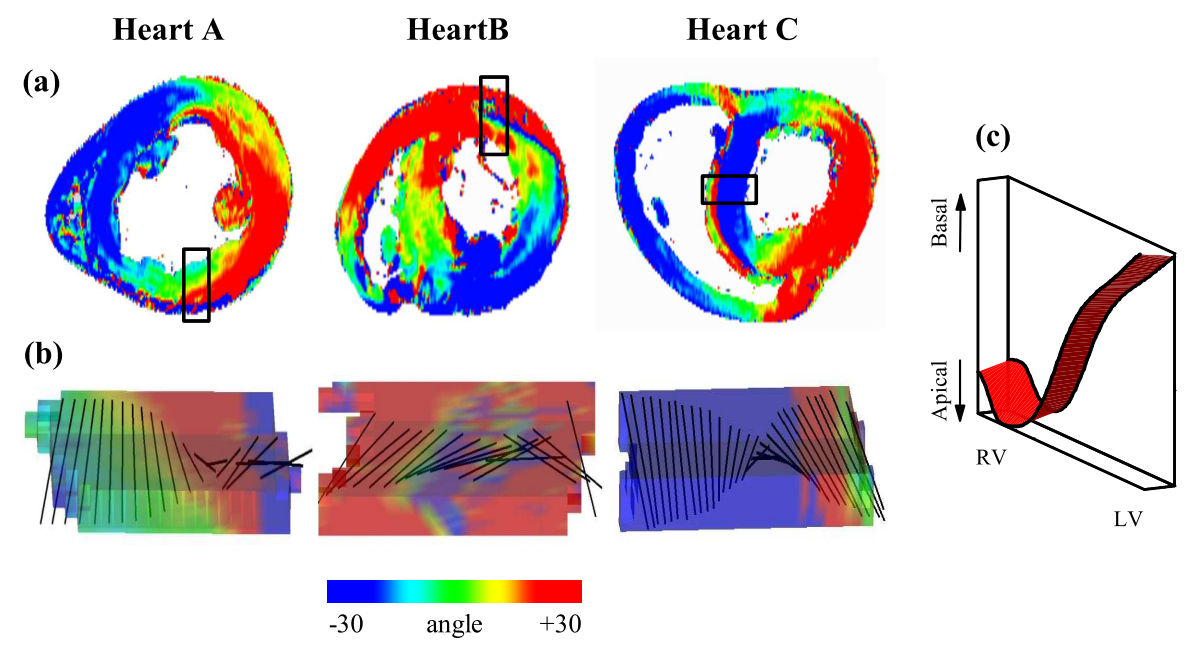

Figure 11: Fibre orientation across transitions between positive and negative orientated cardiac sheets is shown for the three hearts. (a) Three slabs showing abrupt transitions between positive and negative sheet angle were selected (indicated by black boxes) in slices near the equatorial region. These were in the anterior wall for heart A, the posterior wall for heart $\mathrm{B}$ and the septum for heart C. The slices are coloured by sheet angle (see key) with angles more negative than $-30^{\circ}$ coloured blue and angles more positive than $+30^{\circ}$ coloured red. (b) The orientations of the primary eigenvectors (fibre orientations) were visualised in three dimensions in the selected slabs (black lines, superimposed on coloured sheet angle). The fibre orientation shows a smooth transmural rotation from endocardium (left) to epicardium (right), even across the abrupt transition in the sheet angle. (c) Transmural course of the sheet plane reconstructed as in Figure 10 for the septal slab from heart C. The sharp change in sheet angle at $\sim 80 \%$ transmural distance from the left ventricle endocardium is observed as a deflection in the sheet plane. LV, left ventricle; RV, right ventricle. 


\section{Discussion}

\subsection{Fibre Inclination Angle}

The transmural course of the fibre inclination angle in all three hearts follows the classic rotation of fibre orientation with transmural distance, from a positive angle of approximately $60^{\circ}$ in endocardial regions through $120^{\circ}$ to a negative angle of approximately $-60^{\circ}$ in epicardial regions $[51,55,56]$. Our results compare well with previous DTI studies examining the transmural course of the fibre inclination angle [11, 12, 23, 28, 29, 30, 33, 36, 41, 53, 54]. In the equatorial slice, the mean range of the fibre inclination angle was $135^{\circ}$ in the anterior sector, $87^{\circ}$ in the lateral sector, $113^{\circ}$ in the posterior sector, $131^{\circ}$ in the septal sector and $80^{\circ}$ in the LVFW sector. No significant difference was found between the ranges of fibre inclination angles from the five sectors. In other studies, the transmural range of fibre inclination angles was found to be $122-156^{\circ}$ in goat [23], $128-155^{\circ}$ in mouse [41] and $101-134^{\circ}$ in rat [11], and so the range found in canines here compares well with that found in other species.

The slope of the transmural course of the fibre inclination angle ranged from $-109^{\circ}$ in the lateral sector to $-217^{\circ}$ in the anterior sector, with the posterior, septal and RVFW sectors having slopes of $-135,-174$ and $-160^{\circ}$, respectively. No significant difference was found between the slopes from the five sectors. Jiang et al. [41] also found no significant difference in the slope of the fibre inclination angles from different regions in mice, although Geerts et al. [23] found that the lateral and posterior sector slopes in goats $\left(-136\right.$ and $-141^{\circ}$, respectively) were significantly smaller than those from the anterior and septal sectors $(-180$ and $-184^{\circ}$, respectively).

Because of the high resolution of the datasets, the sectors chosen for quantifying fibre inclination angle contain a correspondingly large number of voxels that lie within the ventricular tissue. The three combined anterior sectors of the equatorial slice, for example, contain 1091 voxels, while the lateral, posterior, septal and RVFW sectors contain 879, 896, 675 and 679 voxels, respectively. As far as we are aware, this is the first time such extraction and quantification of DTI data has been undertaken in canine cardiac muscle, and is comparable to the study of goat hearts by Geerts et al. [23]. The variability in fibre inclination angle at a given transmural distance appears to be somewhat larger than in the goat heart study (compare our Figure 7 with Figure 5 in [23]). This variability may be species dependent, with greater variability found in canine than in goat - further work to build libraries of ventricular architecture in numerous species in both physiological and pathological states needs to be undertaken to determine if this is indeed the case.

\subsection{Fibre Transverse Angle}

The transmural course of the fibre transverse angle (Figure 8) shows that, in lateral sectors from equatorial and apical slices, the angle is negative in the endocardial region, changing through to positive in epicardial regions. In basal slices there appears to be no distinct pattern when data from the three hearts are combined. Taking an average value throughout 
the three hearts, we obtained a mean fibre transverse angle of $-2^{\circ}$, which compares well to the mean whole heart value of $-3^{\circ}$ found in goat [23].

\subsection{Sheet Structure}

We chose to characterise the sheet orientation using the secondary eigenvector, which has been validated as corresponding to the local sheet plane [61]. The secondary eigenvector extracted from the diffusion tensor in our datasets represents the sheet plane, as the test statistic $T$ calculated for lateral sectors of basal, equatorial and apical slices all exceeded the critical value $T_{\mathrm{C}}$ (see Table 1 ), and the secondary eigenvalues and corresponding eigenvectors are therefore distinct from the tertiary eigenvalues and eigenvectors.

Recently, Helm et al. [28, 29, 30] using DTI have identified variations in the dominant sheet angles found in different locations of the same heart, especially in basal regions, where the intersection (or sheet) angle in one particular heart ranged from $22^{\circ}$ to $101^{\circ}$. When data from seven hearts were combined, a bimodal distribution of angles was found with two distinct orientations of the cardiac laminar structure lying on average at $45.5^{\circ}$ and $117.6^{\circ}$ [29]. These findings are backed by histological studies $[1,2,11,16,19,27,67]$ that have also identified two distinct populations of sheets in combined data that correlate well with the DTI measurements. Our measured sheet angles for lateral sectors show large intra- and interheart variability, particularly in basal regions (see Figs. 9 and 10). In heart $\mathrm{C}$ for example (circles in Figure 9), the transmural variation in sheet angle in the lateral sector of the basal slice ranges from $-20^{\circ}$ to $+85^{\circ}$, with a change from a value of approximately $-20^{\circ}$ in the subepicardial region to $+80^{\circ}$ in the epicardial region. In endocardial and subendocardial regions, the combined data from the three hearts also shows great variation, ranging from $-30^{\circ}$ in heart A (Figure 9, triangles) to $+85^{\circ}$ in heart $\mathrm{C}$ (Figure 9, circles).

Reconstructions of the transmural course of the sheet plane in lateral sectors are shown in Figure 10, and show that in basal, equatorial and apical slices, the orientation of the sheet plane is, with the exception of the subepicardial and epicardial regions in the apical slices of heart $\mathrm{A}$ and the combined data, in an apex-base direction. Although the selected lateral sectors display qualitatively similar apex-base sheet orientations, Figure 5 shows that there is marked variation in sheet structure between the three hearts. Longitudinal columns of similar sheet orientations extend from apex to base, but the circumferential and transmural locations of these columns and their regions of overlapping and merging varies between the hearts, with hearts $\mathrm{A}$ and $\mathrm{C}$ sharing a predominance of positive sheets antero-laterally and planar sheet anteriorly, negative sheet in the septum, planar sheet posteriorly. Heart B has predominantly negative sheet posteriorly and laterally, planar sheet laterally, negative sheet anteriorly and positive and planar sheet at the septum. Given the greater similarity of hearts $\mathrm{A}$ and $\mathrm{C}$, it is tempting to view heart $\mathrm{B}$ as an aberrant exception. However, heart $\mathrm{B}$ has the closest sheet morphology to that described in LeGrice et al. [46], with positive sheet subendocardially at the septum and negative sheet subendocardially in anterior regions. In addition, the similarity between the sheet structure in hearts $\mathrm{A}$ and $\mathrm{C}$ should not be overstated; the columns of like sheet orientations coincide in transmural location, but the 
pattern of merging of the sheet regions differs markedly. An example of this is at the anterior abutting of the septal negative and lateral positive columns which occurs in both hearts A and C. In the equatorial region of heart A, the negative sheet structure is found subepicardially, and the positive sheet structure deep to this, while in heart B, positive sheets are found subepicardially with negative sheets deep to this (Figure 11a).

Previous quantification of the fibre inclination angle shows a smooth transmural change from approximately $+60^{\circ}$ to $-60^{\circ}$ from epicardium to endocardium. In the light of these data, the sudden switching of sheets orientated at $\sim 70^{\circ}$ to each other [29] is surprising. It might have been anticipated that, as the gross fibre structure crosses over between the two sheet populations, a deviation from the smooth transmural fibre rotation would occur. To further explore the nature of fibre orientation change across the positive-to-negative sheet abutments we reconstructed the three-dimensional orientation of the primary eigenvector across such regions in the three hearts. The visual assessment of the three-dimensional fibre path avoids the limitations associated with reflecting the fibre onto a plane to report a fibre inclination angle [41]. The regions were selected from between the equator and base at the sites of clearly defined sheet abutments (Figure 11a). The fibre reconstruction across the anterior (heart A), posterior (heart B) and septal (heart C) sheet transitions (Figure 11b) show no change in divergence across the abrupt sheet abutment. The transmural course of the sheet plane was reconstructed for the septal sheet transition region in heart $\mathrm{C}$, and shows a divergence in the sheet plane of approximately $70^{\circ}$ (Figure 11c).

This analysis supports the findings of others that there are two populations of sheet angles within the myocardium. Furthermore, it provides further evidence of a switching from positive-to-negative-to-positive sheet orientation as described in references $[13,28,29$, 30]. The data presented here also offer some possible explanations for the differences in the histologically-derived descriptions of sheet morphology in the literature. Some reports $[11,46]$ describe a single population of sheets that bend through the cardiac wall, while others describe dual populations $[1,2,11,16,19,27,67]$. Gross variation in sheet structure between individual hearts will result in differing reports, as will local variation in sheet orientation associated with the precise positioning of the regions selected for characterisation. For an in-depth review on ventricular sheet structure, how sheet structure obtained using DTI and other methods are related, and current and past models of both fibre and sheet structure, we refer the interested reader to reference [24].

\subsection{Limitations}

There are several limitations associated with this study. First, the base-apex axis was fit to the heart manually, as opposed to the mathematical method employed in some other studies (see, for example, reference [23]). However, due to the high resolution of the datasets and the resultant well-defined anatomical landmarks, intra- and inter-observer variability in the fit of the axis was extremely small and resulted in a correspondingly small change $\left(\ll 1^{\circ}\right)$ in calculated fibre and sheet angles. Second, we did not directly compare the fibre and sheet angles obtained using DTI to histological measurements made in the same tissue. However, 
the primary eigenvector of the diffusion tensor has been previously validated as a measure of cardiac fibre orientation $[33,36,53]$, as have the secondary and tertiary eigenvectors with respect to ventricular sheet structure [29]. Nevertheless, combined DTI and histological studies will provide further validation for DTI as a method for obtaining cardiac architecture non-destructively. Third, we do not take into account any potential noise in the diffusion tensor measurements. As stated previously, the DTI method has been validated to provide an accurate measure of cardiac architecture [33, 36, 53, 29], and the interested reader is referred to references $[5,10]$ for further discussion on noise in measured diffusion tensors.

\subsection{Conclusions}

DTI provides a non-destructive, high-throughput, high-resolution method for reconstructing the fibre and sheet orientation throughout cardiac ventricular tissue that can be used to study intra- and inter-heart variations in cardiac architecture, or as the orthotropically anisotropic geometry for computational simulations. Our measurements of the fibre inclination angle in canines correspond well with previous histologically- and DTI-derived measurements. The measured fibre transverse angle and sheet angles, however, show significant inter-heart variability, particularly in basal slices. In addition, the pattern of merging of the two qualitatively different populations of sheet structure (positive and negative) appears to be complex, and the characterisation of any distinct patterns or continuum of patterns within a population [24] will require further work, perhaps using a combination of DTI and histological techniques. We conclude that a single DTI-derived "atlas" model of ventricular fibre and sheet orientation should be applicable to modelling propagation in wedges taken from the equatorial and apical left ventricle, and allow comparisons to experimental studies carried out in wedge preparations. However, due to variability in basal regions of the left ventricles, a library of individual DTI models of basal wedges or of the whole ventricles will be required.

\section{Acknowledgements}

We thank Prof. Edward Hsu for provision of the DTI datasets. This work was supported by the European Union through the BioSim Network of Excellence (contract no. LSHB-CT2004-005137). APB and SHG were supported by the Medical Research Council (UK), PL by the Overseas Research Students Award Scheme.

\section{References}

[1] T. Arts, K.D. Costa, J.W. Covell, A.D. McCulloch. Relating myocardial laminar architecture to shear strain and muscle fiber orientation. Am. J. Physiol., 280 (2001), H2222-H2229. 
[2] H. Ashikaga, J.C. Criscione, J.H. Omens, J.W. Covell. Transmural left ventricular mechanics underlying torsional recoil during relaxation. Am. J. Physiol., 286 (2004), H640H647.

[3] P.J. Basser, J. Mattiello, D. LeBihan. Estimation of the effective self-diffusion tensor from the NMR spin echo. J. Magn. Reson. B, 103 (1994), 247-254.

[4] P.J. Basser, J., Mattiello, D. LeBihan. MR diffusion tensor spectroscopy and imaging. Biophys. J., 66 (1994), 259-267.

[5] P.J. Basser, S. Pajevic. Statistical artefacts in diffusion tensor MRI (DT-MRI) caused by background noise. Magn. Reson. Med., 44 (2000), 41-50.

[6] P.J. Basser, C. Pierpaoli. Microstructural and physiological features of tissues elucidated by quantitative-diffusion-tensor MRI. J. Magn. Reson. B, 111 (1996), 209-219.

[7] A.P. Benson, O.V. Aslanidi, H. Zhang, A.V. Holden. The canine virtual ventricular wall: a platform for dissecting pharmacological effects on propagation and arrhythmogenesis. Prog. Biophys. Mol. Biol., 97 (2008), 187-208.

[8] A.P. Benson, G. Halley, P. Li, W.C. Tong, A.V. Holden. Virtual cell and tissue dynamics of ectopic activation of the ventricles. Chaos, 17 (2007), 015105.

[9] A.P. Benson, A.V. Holden. Effects of geometry and architecture on re-entrant scroll wave dynamics in human virtual ventricular tissues. Lect. Notes Comp. Sci., 4466 (2007), 200-209.

[10] B. Chen, E.W. Hsu. Noise removal in magnetic resonance diffusion tensor imaging. Magn. Reson. Med., 54 (2005), 393-407.

[11] J. Chen, W. Liu, H. Zhang, L. Lacy, X. Yang, S.-K. Song, S.A. Wickline, X. Yu. Regional ventricular wall thickening reflects changes in cardiac fiber and sheet structure during contraction: quantification with diffusion tensor MRI. Am. J. Physiol., 289 (2005), H1898-H1907.

[12] J. Chen, S.-K. Song, W. Liu, M. McLean, J.S. Allen, J. Tan, S.A. Wickline, X. Yu. Remodelling of cardiac fiber structure after infarction in rats quantified with diffusion tensor MRI. Am. J. Physiol., 285 (2003), H946-H954.

[13] A. Cheng, F. Langer, F. Rodriguez, J.C. Criscione, G.T. Daughters, D.C. Miller, N.B. Ingels. Transmural sheet strains in the lateral wall of the ovine left ventricle. Am. J. Physiol., 289 (2005), H1234-H1241.

[14] R.H. Clayton, A.V. Holden. Dynamics and interaction of filaments in a computational model of re-entrant ventricular fibrillation. Phys. Med. Biol., 47 (2002), 1777-1792. 
[15] R.H. Clayton, A.V. Holden. Propagation of normal beats and re-entry in a computational model of ventricular cardiac tissue with regional differences in action potential shape and duration. Prog. Biophys. Mol. Biol., 85 (2004), 473-499.

[16] K.D. Costa, Y. Takayama, A.D. McCulloch, J.W. Covell. Laminar fiber architecture and three-dimensional systolic mechanics in canine ventricular myocardium. Am. J. Physiol., 276 (1999), H595-H607.

[17] L.M. Davis, H.L. Kanter, E.C. Beyer, J.E. Saffitz. Distinct gap junction protein phenotypes in cardiac tissues with disparate conduction properties. J. Am. Coll. Cardiol., 24 (1994), 1124-1132.

[18] J.M. de Bakker, M. Stein, H.V. van Rijen. Three-dimensional anatomic structure as substrate for ventricular tachycardia/ventricular fibrillation. Heart Rhythm, 2 (2005), $777-779$.

[19] S. Dokos, B.H. Smaill, A.A. Young, I.J. LeGrice. Shear properties of passive ventricular myocardium. Am. J. Physiol., 283 (2002), H2650-H2659.

[20] J. Dou, T.G. Teese, W.-Y.I. Tseng, V.J. Wedeen. Cardiac diffusion MRI without motion effects. Magn. Reson. Med., 48 (2002), 105-114.

[21] I.R. Efimov, V.P. Nikolski, G. Salama. Optical imaging of the heart. Circ. Res., 95 (2004), 21-33.

[22] F. Fenton, A. Karma. Vortex dynamics in three-dimensional continuous myocardium with fiber rotation: Filament instability and fibrillation. Chaos, 8 (1998), 20-47.

[23] L. Geerts, P. Bovendeerd, K. Nicolay, T. Arts. Characterization of the normal cardiac myofiber field in goat measured with MR-diffusion tensor imaging. Am. J. Physiol., 283 (2003), H139-H145.

[24] S.H. Gilbert, A.P. Benson, P. Li, A.V. Holden. Regional localisation of left ventricular sheet structure: integration with current models of cardiac fibre, sheet and band structure. Eur. J. Cardiothorac. Surg., 32 (2007), 231-249.

[25] P. Gosse, P. Left ventricular hypertrophy - the problem and possible solutions. J. Int. Med. Res., 33 (2005), 3A-11A.

[26] V. Gulani, G.A. Iwamoto, H. Jiang, J.S. Shimony, A.G. Webb, P.C. Lauterbur. A multiple echo pulse sequence for diffusion tensor imaging and its application in excised rat spinal cords. Magn. Reson. Med., 38 (1997), 868-873.

[27] K.B. Harrington, F. Rodriguez, A. Cheng, F. Langer, H. Ashikaga, G.T. Daughters, J.C. Crisione, N.B. Ingels, D.C. Miller. Direct measurement of transmural laminar architecture in the anterolateral wall of the ovine left ventricle: new implication for wall thickening mechanics. Am. J. Physiol., 288 (2005), H1324-H1330. 
[28] P. Helm, M.F. Beg, M.I. Miller, R.L. Winslow. Measuring and mapping cardiac fiber and laminar architecture using diffusion tensor MR imaging. Ann. N. Y. Acad. Sci., 1047 (2005), 296-307.

[29] P.A. Helm, H.-J. Tseng, L. Younes, E.R. McVeigh, R.L. Winslow. Ex vivo 3D diffusion tensor imaging and quantification of cardiac laminar structure. Magn. Reson. Med., 54 (2005), 850-859.

[30] P.A. Helm, L. Younes, M.F. Beg, D.B. Ennis, C. Leclercq, O.P. Faris, E. McVeigh, D. Kass, M.I. Miller, R.L. Winslow. Evidence of structural remodelling in the dyssynchronous failing heart. Circ. Res., 98 (2006), 125-132.

[31] A.V. Holden, O.V. Aslanidi, A.P. Benson, R.H. Clayton, G. Halley, P. Li, W.C. Tong. The virtual ventricular wall: a tool for exploring cardiac propagation and arrhythmogenesis. J. Biol. Phys., 32 (2006), 355-368.

[32] A.V. Holden, V.N. Biktashev. Computational biology of propagation in excitable media models of cardiac tissue. Chaos, Solitons and Fractals, 13 (2002), 1643-1658.

[33] A.A. Holmes, D.F. Scollan, R.L. Winslow. Direct histological validation of diffusion tensor MRI in formaldehyde-fixed myocardium. Magn. Reson. Med., 44 (2000), 157161.

[34] D.A. Hooks, M.L. Trew, B.J. Caldwell, G.B. Sands, I.J. Legrice, B.H. Smaill. Laminar arrangement of ventricular myocytes influences electrical behavior of the heart. Circ. Res., 101 (2007), e103-e112.

[35] E.W. Hsu, C.S. Henriquez. Myocardial fibre orientation mapping using reduced encoding diffusion tensor imaging. J. Cardiovasc. Mag. Res., 3 (2001), 339-347.

[36] E.W. Hsu, A.L. Muzikant, S.A. Matulevicius, R.C. Penland, C.S. Henriquez. Magnetic resonance myocardial fiber-orientation mapping with direct histological correlation. Am. J. Physiol., 274 (1998), H1627-H1634.

[37] E.W. Hsu, L.A. Setton. Diffusion tensor microscopy of the intervertebral disc anulus fibrosus. Magn. Reson. Med., 41 (1999), 992-999.

[38] P.J. Hunter, P.M.F. Nielsen, B.H. Smaill, I.J. LeGrice I.W. Hunter. An anatomical heart model with applications to myocardial activation and ventricular mechanics. In T.C. Pilkington, B. Loftis, J.F. Thompson, S.L.-Y. Woo, T.C. Palmer, T.F. Budinger (Eds.) High-Performance Computing in Biomedical Research, CRC Press, Boca Raton, USA, 1993.

[39] P.J. Hunter, B.H. Smaill, P.M.F. Nielsen, I.J. LeGrice. A mathematical model of cardiac anatomy. In A.V. Panfilov, A.V. Holden (Eds.) Computational Biology of the Heart, John Wiley and Sons, Chichester, UK, 1997. 
[40] B.A. Inglis, L. Yang, E.D. Wirth, P. Plant, T.H. Mareci. Diffusion anisotropy in excised normal rat spinal cord measured by NMR microscopy. Magn. Reson. Imaging, 15 (1997), 441-450.

[41] Y. Jiang, K. Pandya, O. Smithies, E.W. Hsu. Three-dimensional diffusion tensor microscopy of fixed mouse hearts. Magn. Reson. Med., 52 (2004), 453-460.

[42] P.-S. Jouk, Y. Usson, G. Michalowicz, L. Grossi. Three-dimensional cartography of the pattern of the myofibres in the second trimester fetal human heart. Anat. Embryol., 202 (2000), 103-118.

[43] A.G. Kléber, Y. Rudy. Basic mechanisms of cardiac impulse propagation and associated arrhythmias. Physiol. Rev., 84 (2004), 431-488.

[44] D. LeBihan, J.-F. Mangin, C. Poupon, C.A. Clark, S. Pappata, N. Molko, H. Chabriat. Diffusion tensor imaging: concepts and applications. J. Magn. Reson. Imaging, 13 (2001), 534-546.

[45] I. LeGrice, P. Hunter, A. Young, B. Smaill. The architecture of the heart: a data-based model. Philos. Trans. R. Soc. Lond. A, 359 (2001), 1217-1232.

[46] I.J. LeGrice, B.H. Smaill, L.Z. Chai, S.G. Edgar, J.B. Gavin, P.J. Hunter. Laminar structure of the heart: ventricular myocyte arrangement and connective tissue architecture in the dog. Am. J. Physiol., 269 (1995), H571-H582.

[47] R. Mazhari, J.H. Omens, J.W. Covell, A.D. McCulloch. Structural basis of regional dysfunction in acutely ischemic myocardium. Cardiovasc. Res., 47 (2000), 284-293.

[48] R.J. Myerburg, K.M. Kessler, A. Castellanos. Sudden cardiac death. Structure, function, and time-dependence of risk. Circulation, 85 (1992), I2-I10.

[49] D. Noble, Y. Rudy. Models of cardiac ventricular action potentials: iterative interaction between experiment and simulation. Phil. Trans. Roy. Soc. Lond. A, 359 (2001), 11271142.

[50] C. Pierpaoli, P. Jezzard, P.J. Basser, A. Barnett, G. DiChiro. Diffusion tensor MR imaging of the human brain. Radiology, 201 (1996), 637-648.

[51] J. Ross, E.H. Sonnenblick, R.R. Taylor, H.M. Spotnitz, J.W. Covell. Diastolic geometry and sarcomere lengths in the chronically dilated canine left ventricle. Circ. Res., 28 (1971), 49-61.

[52] J.E. Saffitz, M.D. Lloyd, B.J. Darrow, H.L. Kanter, J.G. Laing, E.C. Beyer. The molecular basis of anisotropy: role of gap junctions. J. Cardiovasc. Electrophysiol., 6 (1995), 498-510. 
[53] D.F. Scollan, A. Holmes, R.L. Winslow, J. Forder. Histological validation of myocardial microstructure obtained from diffusion tensor magnetic resonance imaging. Am. J. Physiol., 275 (1998), H2308-H2318.

[54] D.F. Scollan, A. Holmes, J. Zhang, R.L. Winslow. Reconstruction of cardiac ventricular geometry and fiber orientation using magnetic resonance imaging. Ann. Biomed. Eng., 28 (2000), 934-944.

[55] D.D. Streeter. Gross morphology and fiber geometry of the heart. In R.M. Berne, N. Sperelakis, S.R. Geiger (Eds.) Handbook of Physiology, Section 2: The Cardiovascular System, Volume 1: The Heart, American Physiological Society, Bethesda, USA, 1979.

[56] D.D. Streeter, H.M. Spotnitz, D.P. Patel, J. Ross, E.H. Sonnenblick. Fiber orientation in the canine left ventricle during diastole and systole. Circ. Res., 24 (1969), 339-347.

[57] K.H.W.J. ten Tusscher, O. Bernus, R. Hren, A.V. Panfilov. Comparison of electrophysiological models for human ventricular cells and tissues. Prog. Biophys. Mol. Biol., 90 (2006), 326-345.

[58] R.K. Thakur, G.J. Klein, C.A. Sivaram, M. Zardini, D.E. Schleinkofer, H. Nakagawa, R. Yee, W.M. Jackman. Anatomic substrate for idiopathic left ventricular tachycardia. Circulation, 93 (1996), 497-501.

[59] M.L. Trew, B.J. Caldwell, G.B. Sands, D.A. Hooks, D.C.-S. Tai, T.M. Austin, I.J. LeGrice, A.J. Pullan, B.H. Smaill. Cardiac electrophysiology and tissue structure: bridging the scale gap with a joint measurement and modelling paradigm. Exp. Physiol., 91 (2006), 355-370.

[60] N. Tribulova, S. Novakova, A. Macsaliova, S. Sass, S. Thomas, S. Goetzfried, T. Podzuweit, M. Manoach. Histochemical and ultrastructural characterisation of an arrhythmogenic substrate in ischemic pig heart. Acta Histochem., 104 (2002), 393-397.

[61] W.-Y.I. Tseng, V.J. Wedeen, T.G. Reese, R.N. Smith, E.F. Halpern. Diffusion tensor MRI of myocardial fibers and sheets: correspondence with visible cut-face texture. J. Magn. Reson. Imaging, 17 (2003), 31-42.

[62] M. Valderrábano, P.-S. Chen, S.-F. Lin. Spatial distribution of phase singularities in ventricular fibrillation. Circulation, 108 (2003), 354-359.

[63] F.J. Vetter, A.D. McCulloch. Three-dimensional analysis of regional cardiac function: a model of rabbit ventricular anatomy. Prog. Biophys. Mol. Biol., 69 (1998), 157-183.

[64] M. Vinson, A. Pertsov, J. Jalife. Anchoring of vortex filaments in 3D excitable media. Physica D, 72 (1993), 119-134. 
[65] S.A. Wickline, E.D. Verdonk, A.K. Wong, R.K. Shepard, J.G. Miller. Structural remodeling of human myocardial tissue after infarction. Quantification with ultrasonic backscatter. Circulation, 85 (1992), 259-268.

[66] K. Willecke, J. Eiberger, J. Degen, D. Eckhardt, A. Romualdi, M. Guldenagel, U. Deutsch, G. Sohl. Structural and functional diversity of connexion genes in the mouse and human genome. Biol. Chem., 383 (2002), 725-737.

[67] A.A. Young, I.J. LeGrice M.A. Young, B.H. Smaill. Extended confocal microscopy of myocardial laminae and collagen network. J. Microsc., 192 (1998), 139-150. 